\title{
Road classification based on SVM and vehicle body vibration
}

\author{
Xiaoyi $\mathrm{Fu}^{*}$, and Yuzhuang Zhao \\ Beijing Institute of Technology, Haidian, Beijing, China
}

\begin{abstract}
Keywords: support vector machine, road surface recognition, vehicle information.

Abstract. Obtaining road surface information to make the vehicle run in the best condition can not only reduce energy consumption and vehicle loss, but also improve driving safety. In this paper, specific car body information was preprocessed as root mean square value, and SVM offline training was used. The recognition rate of off-road and highway can reach 98\%. Compared with traditional threshold recognition, SVM has better adaptability and robustness. On the premise of keeping easy to obtain, the discrimination accuracy of the root mean square value is obviously better than the original value and the mean value.
\end{abstract}

\section{Introduction}

With the vigorous development of electronic devices and network technology, the research on vehicles has gradually changed from internal factors like different components to various human-vehicle-environment connections. Humans are drivers, and different drivers have different driving styles, and different emotions will have obvious operating differences, which will affect the driving of the vehicle. Environment refers to other vehicles, road signs, electromagnetic signals, road information, etc., which are transmitted to vehicles as feedback. For the research of unmanned vehicles and smart vehicles, it is obvious that the relationship between the vehicle and the environment is more worthy of in-depth study. Road traffic safety has always been a hot spot that the country attaches great importance to, and is closely related to the safety of people's lives and property. Various collisions are the main form of traffic accidents. Therefore, the quality of road surfaces greatly affects traffic safety. The research on road quality is helpful to improve the understanding of the research concept of automobile technology, so as to design the automobile technology for different road conditions, which can reduce the transportation consumption of the automobile and increase the fatigue life of the automobile. Meantime, it also helps prevent traffic problems in advance. Road data collection tools include millimeter-wave radar, lidar, mono and binocular cameras, and other current advanced vehicle sensors. Since this article is mainly aimed at the identification of road undulations, only simple body pose sensors are needed. Easy accessibility can also increase the commercial value of data.

\footnotetext{
* Corresponding author: lin-ymf@outlook.com
} 


\section{Feasibility analysis}

The data output frequency of the body attitude sensor is $100 \mathrm{~Hz}$, and the dynamic deflection data of the suspension is drawn as shown in Fig. 1.
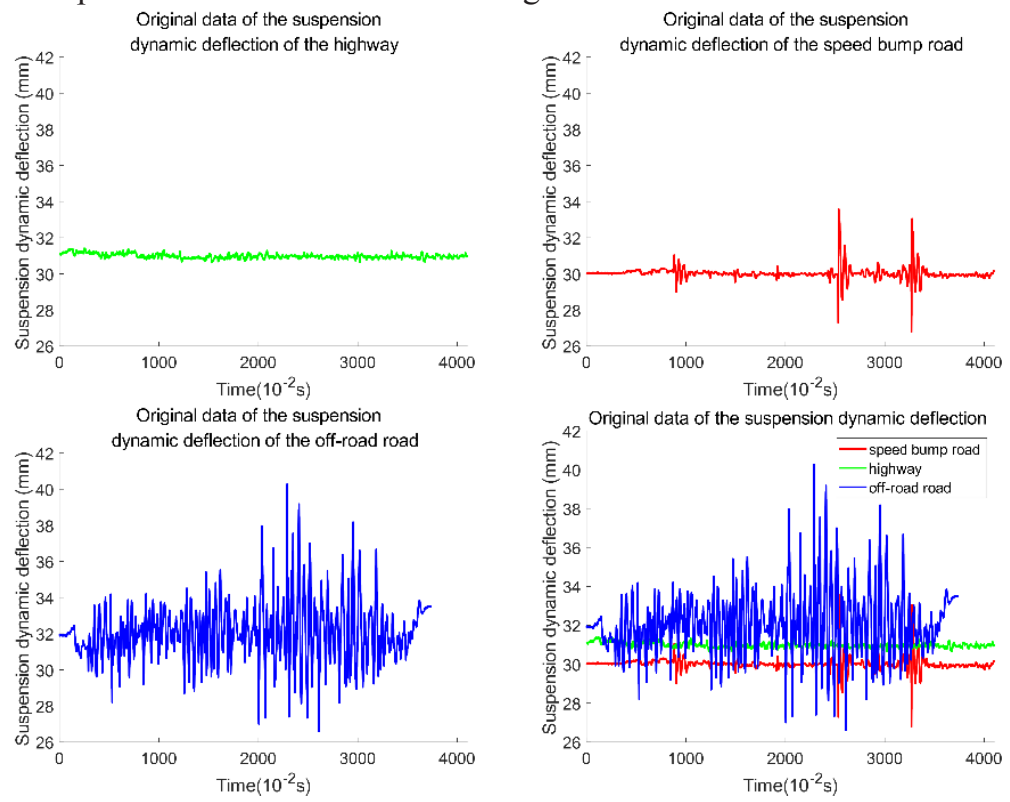

Fig. 1. Original data of suspension dynamic deflection.

It can be seen that the dynamic deflection always fluctuates near a certain constant, that is, the static vehicle height, and the test vehicle is adjustable in height, so the average values of different road surfaces shown in Fig. 1 have obvious differences. Therefore, in order to improve the recognition accuracy and efficiency, the vehicle height value should be eliminated from the suspension dynamic deflection. Similarly, the acceleration of the center of mass also needs to eliminate the interference of the gravity acceleration. In addition, due to the insensitivity of the selected parameters to the direction, considering the use of the root-mean-square value of the statistics, the total amount uses 100 as 1 group and 500 as 1 group, and each replacement amount is 100 numbers, the calculation formulas are (1) and (2) respectively.

$$
\begin{array}{r}
d_{i}=\sqrt{\frac{\sum_{1+100(i-1)}^{100 i} a_{j}^{2}}{100}} . \\
d_{i}=\sqrt{\frac{\sum_{1+100(i-1)}^{400+10 i} a_{j}^{2}}{500}} .
\end{array}
$$

Among them, $a_{i}$ is the real vehicle sensor value collected at $100 \mathrm{~Hz}$, and $d_{i}$ is the sample value required by SVM. For (2), the data has $80 \%$ repeatability.

Fig. 2, Fig. 3, Figure 4 are the results of drawing the root mean square values of specific vehicle parameters under different road surfaces. The left side is the root mean square of 100 numbers, and the right side is the root mean square of 500 numbers. Both The change trend is the same, and the fluctuation of the latter is softer than that of the former. First of all, no matter what conditions are observed, there are huge differences in the shape and value of the curves of highway and off-road roads, which are easy to distinguish. The speed bump pavement is a special type of road, which represents the road with short and drastic changes in the road surface, such as the sunken well surface, the falling rocks on the hillside, and the speed bump. The driving conditions of the car should always be 
maintained in the highway mode. However, in the sudden change of the road, the image using the $1 \mathrm{~s}$ root mean square value is very similar to that of off-road. If misjudgment occurs, the vehicle operation mode will be frequently switched, causing unnecessary loss. When the $5 \mathrm{~s}$ root mean square value is used, the impulse on the road surface of the deceleration zone changes from a "triangular wave" to a "rectangular wave", and off-road roads still have sharp peaks due to the severe turbulence of the road surface.
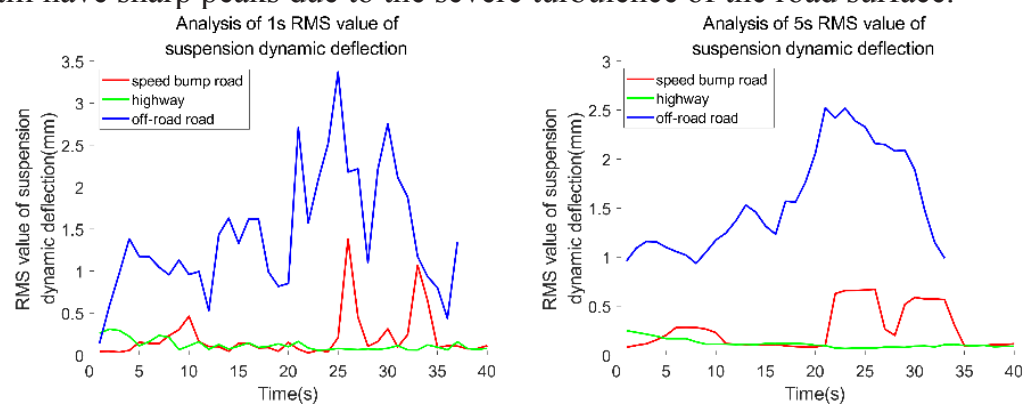

Fig. 2. RMS value of suspension dynamic deflection.
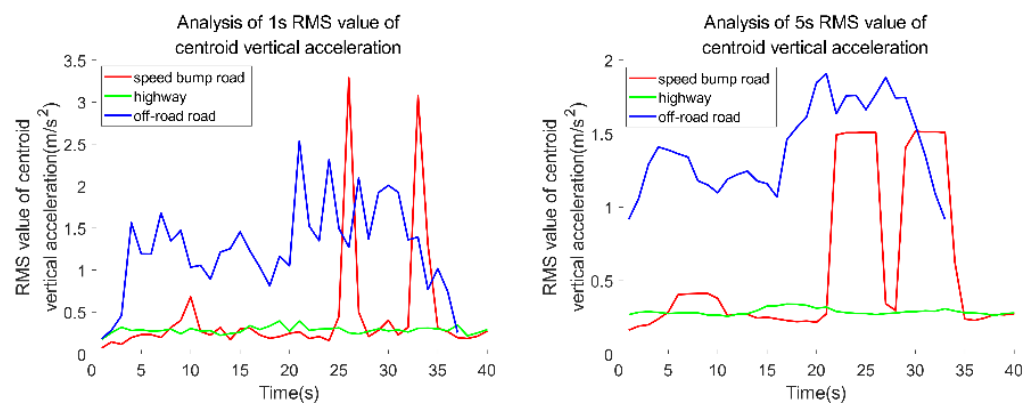

Fig. 3. RMS value of centroid vertical acceleration.
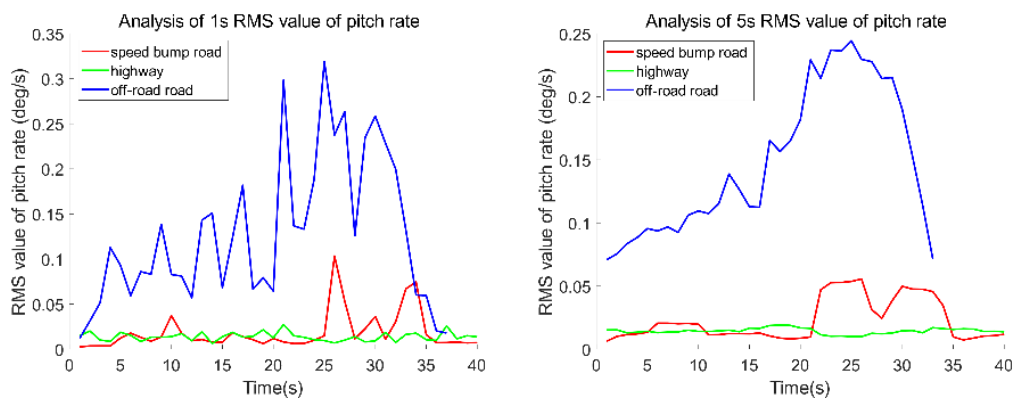

Fig. 4. RMS value of pitch rate.

It can be known from the image that the root mean square value of the body attitude can be used as the identification condition of highway and off-road roads. If the identification system is manually designed, the road surface can be distinguished by the combination of threshold and observation window. For example, if the $1 \mathrm{~s}$ root mean square value of the pitch angular velocity is greater than $0.1(\mathrm{deg} / \mathrm{s})$ for $10 \mathrm{~s}$, it is judged as an off-road road, if the $1 \mathrm{~s}$ root mean square value of the pitch angular velocity is less than $0.05(\mathrm{deg} / \mathrm{s})$ for $10 \mathrm{~s}$, it is judged as a highway. Or the change of the $5 \mathrm{~s}$ root mean square value is for $3 \mathrm{~s}$ greater than $0.01(\mathrm{deg} / \mathrm{s})$ and it is judged as cross-country road, and it is judged as highway for $5 \mathrm{~s}$ less than $0.01(\mathrm{deg} / \mathrm{s})$. It can be seen that it is feasible to use simple data such as body posture to realize road recognition. At the same time, in order to improve the recognition 
speed and accuracy, the SVM machine learning method is used to generate the recognition system.

\section{Recognition process}

In 1995, the famous scholar V. N. VAPNIK proposed the numerical theory of support vector machines, which is based on the VC dimension theory of statistics and a machine theory calculation method under the principle of minimal structural risk.

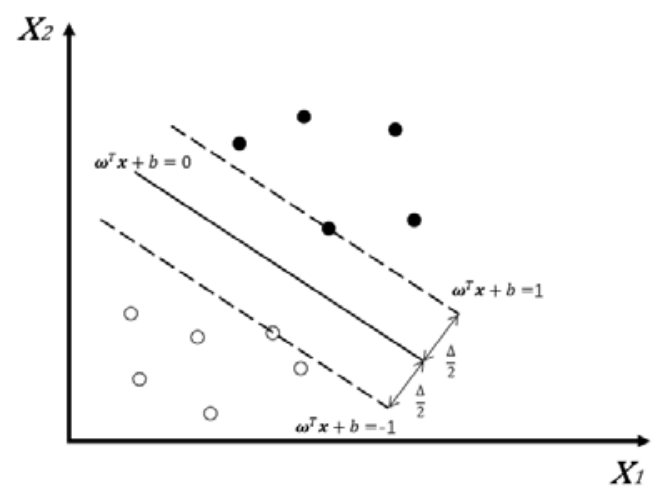

Fig. 5. Two-element classification.

The actual process is to use the kernel function to map the low-dimensional linear inseparable data to the high-dimensional space to solve the convex quadratic programming, and obtain a hyperplane to separate the data. For example, in the binary classification problem shown in Fig. 5, the solid circle is the positive class $\{x, 1\}$ and the hollow circle is the negative class $\{\mathrm{x},-1\}$, then this set of data is linearly divided by a straight line (hyperplane).

$$
\vec{\omega}^{T} \vec{x}+b=0 .
$$

Obviously, there are countless straight lines that can separate this set of data, and the optimal solution we are looking for is the hyperplane when $\Delta$ is the largest, and the distance between the two dotted lines is the maximum distance.

$$
d=\frac{2}{\|\vec{\omega}\|}
$$

Finally, it is reduced to the optimization problem under the inequality constraint shown in equation (5).

$$
\left\{\begin{array}{c}
\min \frac{1}{2}\|\vec{\omega}\|^{2} \\
\text { s.t. } y_{i}\left(\vec{\omega}^{T} \vec{x}_{i}+b\right) \leq 0
\end{array} .\right.
$$

Solving the above equation requires the use of Lagrange multiplier method under KKT conditions. For most realistic problems that are linearly inseparable, SVM needs to introduce slack variables and kernel functions to turn them into high-dimensional linearly separable solutions.

The programming environment of this experiment is MATLAB2018a, using the SVM pattern recognition and regression software package developed and designed by Professor Lin Chih-Jen of Taiwan University, and using cross-validation to find the best radial basis function parameters $\gamma$ and loss function $\mathrm{C}$. 
When the sensor data type is insufficient due to other factors such as economy or structure, a single data should be used to realize road recognition. Since a single data has no learning significance for the machine, and three data types are selected in this experiment, in order to form a better comparison effect, under the condition of a single data, three data are also taken as a set of simulated three-dimensional. Therefore, you can set sampling at $100 \mathrm{~Hz}$, calculate the root mean square value of the data every 1 second, and use the three root mean square values of the first 3 seconds every 1 second, that is, the data repeatability is $66 \%$. The advantage of this selection is that it can ensure the timeliness of identification, avoid slow identification due to data generation, and at the same time, continuous data can improve the correlation of identification. The disadvantage is that occasional abnormal data has a greater impact.

Table 1. Highway-off-road experiment using centroid vertical acceleration.

\begin{tabular}{c|c|c|c|c|c}
\hline $\begin{array}{c}\text { Experiment } \\
\text { number }\end{array}$ & 1 & 2 & 3 & 4 & 5 \\
\hline Accuracy & $94.6 \%$ & $98.2 \%$ & $98.2 \%$ & $96.4 \%$ & $100 \%$ \\
\hline $\begin{array}{c}\text { Error } \\
\text { number }\end{array}$ & $36,37,70$ & 36 & 36 & 36,70 & 1 \\
\hline
\end{tabular}

The results of the five experiments based on the centroid acceleration root mean square value are shown in Table 1 . There are 70 sets of data. The first 35 sets correspond to highway surfaces, and the last 35 sets are off-road roads. Randomly select 14 groups as training samples and 56 groups as identification samples. It can be seen that the accuracy rate is almost maintained above $95 \%$. At the same time, most cases of wrong judgment are judged to be a highway road from off-road roads when the vehicle starts and stops.

Table 2. Speed bump road-off-road experiment using centroid vertical acceleration.

\begin{tabular}{c|c|c|c|c|c}
\hline $\begin{array}{c}\text { Experiment } \\
\text { number }\end{array}$ & 1 & 2 & 3 & 4 & 5 \\
\hline Error & $24,25,26$, & $24,25,26$, & $24,25,26,31,32$, & $24,25,26$, & $24,25,26$, \\
number & 32,33 & $31,32,33$ & 33,34 & $31,32,33$ & $31,32,33$ \\
\hline
\end{tabular}

In the comparison experiment between speed bumps road and off-road road, if the speed bump road is selected as the training sample, the training time of the learning machine will be too long due to the special data of the speed bump. And the type of pavement is determined by the quality and shape of the pavement, so the training sample still selects the general road sample. The five results are shown in Table 2. In order to include the complete speed bump response, 40 sets of speed bump samples are taken for identification, and the error number in the table is just the data of the vehicle passing the speed bump, which is mistakenly judged as an off-road road.

In the same way, when using suspension dynamic deflection and pitch angle speed for highway and off-road identification, in addition to misjudgments when starting and braking, there will also be misjudgments in individual driving positions in the middle. Their identification stability is slightly worse than that of the "centroid accelerometer", and may get $100 \%$ accuracy, or it may be as low as $88 \%$.

Table 3. Highway-off-road experiment using multidimensional data.

\begin{tabular}{c|c|c|c|c|c}
\hline $\begin{array}{c}\text { Experiment } \\
\text { number }\end{array}$ & 1 & 2 & 3 & 4 & 5 \\
\hline Accuracy & $96.4 \%$ & $98.2 \%$ & $98.2 \%$ & $94.6 \%$ & $98.2 \%$ \\
\hline $\begin{array}{c}\text { Error } \\
\text { number }\end{array}$ & 36,37 & 36 & 36 & $36,37,38$ & 36 \\
\hline
\end{tabular}

Through the feasibility analysis, it can be seen that the vertical acceleration of the center of mass, the suspension dynamic deflection, and the pitch angular velocity have a strong 
correlation, so a three-dimensional variable can be composed of the data per second. Although no new car body information has been added, the influence of special data will decrease due to the independence of the data per second. Moreover, the sensitivity of the three types of data is different, which can improve the misjudgment of certain impacts. Before support vector machine recognition, due to different data types, normalization processing is required. Before support vector machine recognition, due to different data types, normalization processing is required. As shown in Table 3, in the highway-off-road identification model that uses the three data at the same time, the accuracy rate still maintains a high level of over $95 \%$, and the misjudgment at braking is significantly reduced.

Table 4. Speed bump road-off-road experiment using multidimensional data.

\begin{tabular}{c|c|c|c|c|c}
\hline $\begin{array}{c}\text { Experiment } \\
\text { number }\end{array}$ & 1 & 2 & 3 & 4 & 5 \\
\hline $\begin{array}{c}\text { Error } \\
\text { number }\end{array}$ & $26,33,34$ & $26,33,34$ & $26,33,34$ & $10,26,33,34$ & $26,33,34$ \\
\hline
\end{tabular}

In the speed bump-off-road experiment, although the judgment errors of the two speed bumps were still not avoided, it can be seen that the number of the wrong experiment was significantly reduced. The reason is that there is no overlap on the data and no interference with each other. Then under this model, simple post-processing such as combined weighting on the recognition results can eliminate the effect of instantaneous large-scale vibration.

\section{Conclusion}

After a series of comparative tests, it is shown that SVM recognition using the data of centroid vertical acceleration, suspension dynamic deflection, and pitch angular velocity data can effectively distinguish highway roads from off-road roads. For the selection of initial data, two methods are provided, single data and multiple data. The highway-off-road discrimination accuracy of both methods is very high, reaching over $94 \%$. Speed bumps and off-road identification situations failed to correctly handle the speed bump impact, but the result of multi-data processing is less dead pixels, which is convenient for later repair. Therefore, how to optimize can more intelligently distinguish the short-term high-intensity impact when driving smoothly is a direction that can continue to be deepened. Finally, road recognition based on the body posture is a posterior logic, and it needs to travel a certain length of road before it can be recognized. Image recognition can predict the road in advance. Therefore, combining the two will be an economic and simple road identification trend.

\section{References}

1. J.R. Ng, J.S. Wong, V.T. Goh, et al. Identification of Road Surface Conditions using IoT Sensors and Machine Learning: 5th ICCST 2018, Kota Kinabalu, Malaysia, 29-30 August 2018// Computational Science and Technology. (2019).

2. R. He, L. Zhang. He R, Zhang L . Interval Recognition Algorithm of the Pavement Surface Condition Based on Lagrange Interpolation Method[J]. Mathematical Problems in Engineering, 2020, 2020(1):1-8.

3. G. Safont, A. Salazar, A. Rodriguez, et al. Multichannel Signal Processing for Road Surface Identification. IEEE, (2020). 
4. J. Zhao, H. Wu, L. Chen. Road Surface State Recognition Based on SVM Optimization and Image Segmentation Processing. Journal of Advanced Transportation, 1-21, (2017).

5. A. Gain and D. Roy, A novel algorithm for object detection in low contrast image and recognition by SVM, IJSR, 4, (2015).

6. T.S.G. Mendes , P.D.P. Aluir. Urban road classification in geometrically integrated high-resolution RGB aerial and laser-derived images using the artificial neural network classification method. International Journal of Image and Data Fusion, 10(8):1-21 (2018). 